\title{
Diretrizes para o Ensino na Atenção Primária à Saúde na Graduação em Medicina ${ }^{I}$ \\ Guidelines for Primary Health Care Teaching in Undergraduate Medical Education
}

Marcelo Marcos Piva Demarzo ${ }^{1}$, Rodrigo Cariri Chalegre de Almeida², João José Neves Marins², Thiago Gomes da Trindade ${ }^{1}$, Maria Inez Padula Anderson ${ }^{1}$, Airton Tetelbom Stein ${ }^{2}$,

Fabiano Gonçalves Guimarães ${ }^{1}$, Felipe Proenço de Oliveira ${ }^{2}$, Fernanda Plesmann de Carvalho ${ }^{1}$, Flavio Dias Silva ${ }^{1}$, Francisco Arsego de Oliveira², Gustavo Tenório Carlos², Jaciara Bezerra Marques², Leika Aparecida Ishigama Geniole ${ }^{1}$, Lia Márcia Cruz da Silveira ${ }^{1}$, Maria Eugênia Bresolin Pinto ${ }^{2}$, Nayra Almeida da Silva ${ }^{1}$, Rodrigo Cechelero Bagatelli ${ }^{1}$, Sandro Rogério Rodrigues Batista ${ }^{2}$ Tânia de Araújo Barboza ${ }^{1}$, Thiago Dias Sartiㄹ․ Vitor Barreto ${ }^{1}$, Gustavo Diniz Ferreira Gusso ${ }^{1}$, Mourad Ibrahim Belaciano ${ }^{2}$

\footnotetext{
PALAVRAS-CHAVE:

- Educação Médica

- Atenção Primária à Saúde

- Currículo
}

\section{KEYWORDS:}

- Education, Medical

- Primary Health Care

- Curriculum 


\section{INTRODUÇÃO}

Um marco reconhecido na política educacional brasileira é a publicação das Diretrizes Curriculares Nacionais (DCN) ${ }^{1}$, as quais flexibilizam as organizações curriculares, possibilitando a construção de projetos político-pedagógicos (PPP) mais condizentes com o pensamento contemporâneo. A política de saúde no Brasil também experimenta um marco em sua história recente com a Constituição Federal de 1988, que instituiu em todo o território nacional um sistema universal e único de saúde, o Sistema Único de Saúde (SUS). Dentre inúmeras proposições contundentes, a Constituição definiu que a ordenação da formação de recursos humanos também é competência do SUS. A Atenção Primária à Saúde (APS) é ponto de convergência entre estas duas políticas, na medida em que as DCN apontam para uma integração com o sistema de saúde e com as necessidades de saúde da população, descentralizando o ensino da medicina dos hospitais à rede de saúde, tendo, na Estratégia de Saúde da Família (ESF), o modelo prioritário para a (re) organização da APS e toda a atenção à saúde no país.

Neste contexto, a Associação Brasileira de Educação Médica (ABEM) e a Sociedade Brasileira de Medicina de Família e Comunidade (SBMFC) vêm empreendendo esforços conjuntos de produção técnica e pedagógica para apoiar as escolas no processo de mudança (documentos disponíveis nos portais das entidades na Internet: http:/ / www.sbmfc.org.br e http:/ / www.abem-educamed.org.br), com base nas DCN e com foco no ensino da APS na graduação em Medicina. Para a produção das presentes Diretrizes, as entidades examinaram documentos e publicações de pesquisadores nacionais das áreas de Saúde Coletiva e de Educação Médica, além de resoluções nacionais e internacionais para o ensino da APS e da Medicina de Família e Comunidade (MFC), que é entendida como especialidade médica com foco privilegiado na APS.

\section{PERCURSO METODOLÓGICO}

Para construção das Diretrizes, inicialmente, elaborou-se um documento preliminar ${ }^{2}$ numa oficina de trabalho com duração de três dias, com professores e preceptores que atuam na APS e MFC, considerados espertos no tema pelas duas entidades. Com a finalização do documento preliminar em 26 de julho de 2009, iniciou-se uma consulta pública nos portais virtuais das duas entidades, como também nos congressos nacionais: Congresso Brasileiro de Educação Médica (COBEM), de 2009 e 2010, e Congresso Brasileiro de Medicina de Família e Comunidade, de 2009, garantindo assim participação ampla, coletiva e democrática de professores, preceptores, estudantes, gestores e pessoas da comunidade. O presente documento é a síntese do documento preliminar e das sugestões que apareceram na consulta pública, cujo encerramento ocorreu em 29 de outubro de 2010 durante o COBEM.

\section{DIRETRIZES}

Os resultados apresentados estão organizados como: contribuições da APS para a graduação em Medicina ("por quê"); objetos de aprendizado ("o quê"); metodologias e estratégias didáticas ("como"); momentos ("quando"); espaços formativos ("onde") e os principais atores envolvidos ("quem").

\section{“POR QUÊ?" - CONTRIBUIÇÕES DA ATENÇÃO PRIMÁRIA À SAÚDE PARA A GRADUAÇÃO EM MEDICINA}

- O estudante é favorecido ao lidar com diferentes aspectos da vida e seus ciclos, na sua complexidade clínica e cultural.

- É possibilitada ao estudante a atuação em relação ao indivíduo e ao coletivo de forma contextualizada à realidade local.

- Constitui cenário de integração de práticas das diferentes áreas, campos e núcleos de conhecimento (ciências básicas, especialidades médicas e saúde coletiva).

- Contribui para o estudante ter uma compreensão da rede intersetorial de atenção e cuidados em saúde.

- Contribui para o desenvolvimento de uma prática clínica integrada, possibilitando a interdisciplinaridade.

- Favorece o aumento da resolubilidade clínica ao lidar com condições e problemas complexos e singulares de saúde, de forma contínua e longitudinal.

- Contribui para conceituação de saúde e adoecimento, respeitando o saber do outro e da comunidade local.

- Contribui para a concepção de conhecimento dinâmico e em construção, que articule outros conhecimentos e realidades.

- Favorece o desenvolvimento da competência cultural e dialógica na comunicação em saúde.

\section{“O QUÊ?" - OBJETOS DE ENSINO-APRENDIZAGEM}

Entende-se que a ordem cronológica não interfere no desenvolvimento da competência final em APS, desde que as três abordagens constituam eixos estruturantes da formação médica e sejam desenvolvidas ao longo dos seis anos de curso. Ao final do sexto ano, o estudante de Medicina deve ter competência nas três dimensões a seguir, de forma integrada:

Na abordagem individual, deve-se:

- conhecer e utilizar a abordagem clínica centrada na pessoa integral, complexa, interdisciplinar, longitu- 
dinal e resolutiva, utilizando as evidências científicas como ferramenta e suporte, porém, singularizando o processo;

- estabelecer o primeiro contato com os pacientes, lidando com problemas não-selecionados e indiferenciados, reconhecendo as incertezas no cotidiano da prática clínica da APS;

- desenvolver e aplicar a consulta do médico de família e comunidade para promover uma eficaz relação médico-paciente, com respeito pela autonomia deste;

- relacionar os processos específicos de decisão com a prevalência e a incidência das doenças na comunidade;

- reunir e interpretar seletivamente a informação recolhida na anamnese, no exame objetivo e nos exames complementares, e aplicá-la a um plano de ação adequado em colaboração com o paciente;

- manejar simultaneamente múltiplas queixas e patologias, tanto problemas de saúde agudos como crônicos das pessoas;

- promover a saúde e o bem-estar, aplicando adequadamente as estratégias de promoção da saúde e prevenção da doença;

- conciliar as necessidades de cada paciente e as de saúde da comunidade em que ele vive, de acordo com os recursos disponíveis.

$\mathrm{Na}$ abordagem familiar, deve-se: conhecer e lidar com as distintas fases do ciclo vital; conhecer e lidar com a estrutura e dinâmica familiar, utilizando os instrumentos do diagnóstico familiar, como o genograma e ecomapa na abordagem familiar; identificar a influência das relações intrafamiliares no processo de saúde e adoecimento.

$\mathrm{Na}$ abordagem comunitária, deve-se: conhecer e lidar com instrumentos de diagnóstico de saúde da comunidade, acessando os diversos setores relacionados e correlacionando-os com a prática clínica do médico; identificar a organização da sociedade e da comunidade, os modos de produção presentes e os determinantes sociais do processo saúde-adoecimento; identificar e respeitar a diversidade cultural; compreender o que é "território vivo"; reconhecer e desenvolver ações de vigilância em saúde e participar de atividades de educação popular em saúde, compreendendo a existência de diferentes concepções pedagógicas e valorizando o saber popular.

\section{“QUANDO?" - MOMENTOS FORMATIVOS}

Destacam-se as seguintes orientações gerais quanto aos tempos de inserção do ensino da APS nas grades curriculares de Medicina:
- deve ser uma inserção longitudinal e com continuidade ao longo do curso, num modelo em espiral crescente de complexidade e, de preferência, com atividades na APS em todos os períodos (semestre ou anos) do curso;

- devem ser incluídas, desde o primeiro ano, atividades envolvendo as abordagens do indivíduo, da família e da comunidade, de forma integrada;

- devem ser introduzidos módulos, disciplinas, unidades educacionais ou estágios de APS centrados na clínica do médico de família (método clínico centrado na pessoa), nos ciclos e anos intermediários (terceiro e quarto anos e períodos correspondentes);

- as competências (conhecimentos, habilidades e atitudes) adquiridas ao longo dos anos na APS, incluindo os estágios práticos, devem ser mais bem sedimentadas durante o internato (quinto e sexto anos), em estágios de treinamento clínico em serviços de APS, sob supervisão.

\section{“COMO?" - DIRETRIZES METODOLÓGICAS E ESTRATÉGIAS DIDÁTICAS}

Como diretrizes metodológicas e estratégias didáticas, são fundamentais:

- inserção significativa, com objetivos claros de ensino-aprendizagem e cronograma de trabalho, evitando-se apenas estágios de observação; com estratégias que impliquem o estudante na corresponsabilização do cuidado, contribuindo efetivamente para o serviço em que ocorre a prática, construindo projetos terapêuticos em conjunto nas equipes de APS dentro de cada unidade;

- metodologias dialógicas e ativas de ensino-aprendizagem como referência no ensino na APS, integrando prática e teoria, problematizando a realidade e possibilitando a reflexão sobre a prática profissional, trabalhando em pequenos grupos de forma espiral (progressiva) e com diversidade de cenários e atividades;

- favorecimento de ambiente multi e interdisciplinar e atuação conjunta com estudantes e profissionais de outras áreas;

- inserção no contexto do desenvolvimento das habilidades clínicas, com foco no seguimento de pessoas e famílias, desde o início da graduação;

- integração com outras disciplinas do curso de Medicina, possibilitando espaços de troca, tanto ao longo do curso como num semestre específico;

- estímulo para a utilização adequada e contextualizada de condutas clínicas baseadas em evidências científicas, estimulando e aproximando o estudante da 
investigação e produção científica; ao mesmo tempo, singularizando o processo de produção do cuidado, com foco nas pessoas e comunidades;

- pactuação conjunta com as equipes de APS, gestor local e com a comunidade das atividades e ações individuais e coletivas a serem realizadas pelos estudantes;

- incentivo para a utilização de estratégias de ensino à distância e telessaúde;

- busca de integração com pesquisa e extensão;

- utilização de métodos avaliativos formativos, além dos somativos, os quais sejam contextualizados e adequados ao desenvolvimento da competência em APS.

\section{“ONDE?" - ESPAÇOS FORMATIVOS}

Entende-se, como cenários ou equipamentos de APS adequados enquanto espaços formativos para a graduação em Medicina unidades, equipamentos de saúde e redes de apoio social próprios de APS, incluindo o sistema suplementar, desde que possibilitem o desenvolvimento das competências definidas nestas diretrizes, dentro da lógica definida atualmente pela Política Nacional de Atenção Básica (PNAB) ${ }^{3}$, com estrutura adequada para receber atividades de ensino e estudantes; a estrutura adequada deve ser pactuada entre os atores da integração ensino-serviço-gestão e comunidade, com base nas diretrizes de qualidade do Ministério da Saúde e da Educação, levando-se em consideração:

- a existência de um número e tamanho adequados de salas de atendimento individual e coletivo para participação efetiva dos estudantes nas atividades da equipe de APS;

- a existência de espaço físico de estudo e conforto para os estudantes, com acesso ao material bibliográfico próprio da APS;

- a existência de meio de transporte público que permita o acesso adequado dos estudantes às unidades e aos equipamentos de saúde;

- o estabelecimento de instrumentos jurídico-legais firmados entre as Instituições de Ensino Superior (IES) e o gestor municipal, os quais estabeleçam o desenvolvimento de atividades em longo prazo, com a inclusão de colegiados gestores paritários, tendo a participação de estudantes e do controle social;

- Unidades de Saúde da Família ou similares, integradas à rede municipal de saúde, articuladas em todos os níveis do sistema, com estímulo para que as IES participem no desenvolvimento dessa rede;

- preferencialmente, concentrando as atividades em determinado território ou distrito, a fim de permitir melhor acompanhamento, continuidade e contextuali- zação do desenvolvimento das atividades, integrando os diversos equipamentos da rede de atenção à saúde;

- preferencialmente em Unidades de Saúde que tenham Programas de Residência em MFC;

- utilizando equipamentos comunitários enquanto espaços de formação.

\section{“QUEM?" - DOCENTES E PRECEPTORES}

Considerando-se o acúmulo internacional sobre a participação do médico especialista em MFC no ensino da APS, na graduação em Medicina, e respeitando as necessidades e a autonomia de cada IES, é fundamental a participação do médico especialista em MFC (com residência médica ou título de especialista na área) no quadro de docentes e preceptores. Recomenda-se ter uma proporção adequada de docentes especialistas em MFC entre os docentes universitários, para adequar-se ao modelo pedagógico e às necessidades de saúde da população.

Também devem ser criadas vagas docentes, com carga horária adequada, dentro da estrutura universitária de cada IES, as quais garantam a presença do médico especialista em MFC e dos demais profissionais da APS.

O ensino da abordagem clínica individual na APS, principalmente nos anos intermediários do curso e no internato, deve ser realizado pelo médico especialista em MFC, em parceria com outros profissionais clínicos com formação e/ou vivência em tal cenário. A Figura 1 traz uma representação gráfica em " $Z$ ", sugerindo a referência docente para o estudante ao longo dos seis anos do curso médico, no cenário da APS.

$\mathrm{Na}$ abordagem familiar e comunitária, deve haver, além do médico especialista em MFC, outros profissionais com competência em tais abordagens.

Caso não exista no quadro da IES o especialista em MFC, a instituição deve pactuar com os gestores universitários e do sistema de saúde a formação e/ou especialização deste profissional médico.

Deve haver um programa de desenvolvimento profissional contínuo e uma educação permanente para a docência em APS, incluindo docentes e médicos dos serviços (preceptores), desenvolvido pelas IES, em parceria com o gestor local de saúde. O docente da IES deve estar efetivamente inserido nas equipes de APS para acompanhar e supervisionar o processo.

É bom haver uma ótima relação entre o número de preceptores e estudantes em cada equipe de APS, pensando na qualidade da preceptoria.

Deve-se garantir que a agenda de atividades do preceptor nas unidades de saúde seja compatível com as atividades de ensino, assistência e educação permanente. O Quadro 1 sumariza as principais recomendações das Diretrizes. 


\section{FIGURA 1}

Representação esquemática em "Z" da referência docente para o estudante no cenário da Atenção Primária à Saúde, ao longo dos seis anos do curso médico

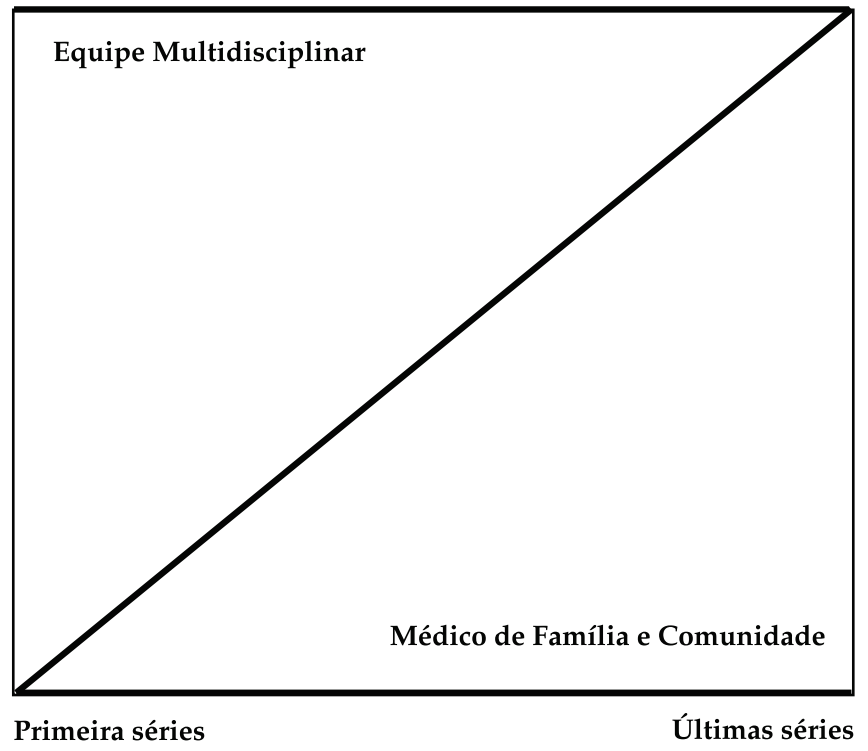

QuAdro 1

Sumário das diretrizes para o ensino na APS na graduação em Medicina

\section{Por que ensinar na APS ?}

- Contribui para o desenvolvimento de uma prática clínica integrada e contextualizada, que é centrada nas pessoas e comunidades, possibilitando a interdisciplinaridade

\section{O que ensinar?}

- Abordagem individual e coletiva, com orientação familiar e comunitária, como eixos estruturantes da formação médica

\section{Quando ensinar?}

- Deve ser uma inserção longitudinal e com continuidade ao longo do curso, em um modelo em espiral crescente de complexidade, e de preferência, com atividades na APS em todos os períodos (semestres ou anos) do curso.

\section{Como ensinar?}

- Com metodologias dialógicas e ativas de ensino-aprendizagem, integrando prática e teoria, problematizando e possibilitando uma reflexão sobre a prática profissional, trabalhando em pequenos grupos e com diversidade de cenários e atividades

\section{Onde ensinar?}

- Em unidades de Saúde da Família, ou similares, integradas à rede municipal, articuladas com a rede de atenção à saúde, com estrutura adequada para o recebimento de estudantes e para um efetivo processo de ensino-aprendizagem.

Quem deve ensinar?

- É fundamental a participação do médico especialista em MFC como docente e preceptor, em parceria com outros profissionais com vivência e competência em APS.

\section{CONSIDERAÇÕES FINAIS}

Este é um documento "vivo", que deve estar em constante processo de atualização e revisão, e que visa, sobretudo, desencadear as discussões sobre o tema. Trata-se de diretrizes construídas com a intenção de apoiar as escolas médicas, de forma objetiva e prática, na elaboração de seus projetos pedagógicos no contexto da APS.

Considera-se que os núcleos do saber e do agir médico se encontram no aprendizado da clínica, mais especificamente no raciocínio clínico e semiológico. Por isso, destaca-se a imperiosidade de que o ensino na APS esteja presente longitudinalmente ao longo do curso, de preferência com inserções significativas (de aprendizado real e a partir do trabalho), mas que, sobretudo, deva fazer parte do núcleo do ensino da semiologia e da prática clínica do futuro médico.

Enfim, tais Diretrizes apontam para a necessidade de outros processos institucionais e políticos nas áreas de educação e saúde para a sua efetivação. Faz-se necessário uma agenda junto aos Ministérios da Saúde e da Educação, como também junto às IES, para que sejam discutidas estratégias de ampliação e renovação do quadro de docentes no Ensino Superior, assim como de qualificação dos serviços e equipamentos de APS, expressos em contratos claros de compartilhamento dos objetos de gestão e ensino, contribuindo para a melhora das condições da graduação em Medicina no Brasil.

\section{AGRADECIMENTOS}

A todos os participantes da consulta pública nos portais virtuais e nos congressos da ABEM e SBMFC, pela disposição e empenho na elaboração destas Diretrizes.

Fonte de financiamento: ABEM e SBMFC.

\section{REFERÊNCIAS}

1. Brasil. Ministério da Educação. Conselho Nacional de Educação. Câmara de Educação Superior. Resolução CNE/ CES nº 4, de 07 de novembro de 2001. Diretrizes Curriculares Nacionais do Curso de Graduação em Medicina. [online]. Brasília, DF; 2001. [acesso em 1 nov. 2010]. Disponível em: http://portal.mec.gov.br/cne/arquivos/ pdf/CES04.pdf

2. Gusso G, Marins JJN, Demarzo MMP, Belaciano MI, Almeida RCC, Stein AT, et al. Diretrizes para o Ensino na Atenção Primária à Saúde na Graduação em Medicina - SBMFC e ABEM. Cad ABEM. 2009 [online]; 5: 13-20. [acesso em 1 nov. 2010]. Disponível em: http://www.abem-educmed. org.br/pdf_caderno5/atencao_primaria_saude.pdf

3. Brasil. Ministério da Saúde. Departamento de Atenção Básica. Política Nacional de Atenção Básica. Brasília: Ministério da Saúde; 2006. 


\section{CONFLITO DE INTERESSES}

Declarou não haver.

\section{ENDEREÇO PARA CORRESPONDÊNCIA}

Marcelo Demarzo

Rua Borges Lagoa, 1341,

Vila Clementino - São Paulo

CEP. 04038-036 SP 